Part IV

Supernovae: Models 


\title{
Models of Supernova Explosions: Where Do We Stand?
}

\author{
Wolfgang Hillebrandt \\ Max-Planck-Institut für Astrophysik, 85748 Garching, Germany; \\ wfh@mpa-garching.mpg.de
}

\begin{abstract}
Summary. The present status of our understanding of core-collapse and of thermonuclear supernovae is reviewed. It will be argued that the failure of numerical simulations of the collapse of massive stars to produce explosions is probably caused by our incomplete knowledge of the (micro-) physics involved. In contrast, for thermonuclear (type Ia) supernovae the basic physics seems to be well under control and, therefore, it is not surprising that model predictions and observations are in good agreement.
\end{abstract}

\section{Introduction}

The modeling of both core-collapse and thermonuclear supernovae is still a major a challenge in astrophysics. In the case of core collapse models, which utilize gravitational binding for the explosion, the problem is not so much an energy but rather a momentum problem. There is plenty of energy available and only a small fraction of it has to be converted into outward momentum of the stellar envelope, but this is a non-trivial problem. It is somewhat embarrassing that despite all the effort that went into the construction of core-collapse supernova models until now none of them yielded the desired explosions.

Thermonuclear explosion models, on the other hand, suffer from the fact that once nuclear burning is ignited the progenitor star, presumably a white dwarf, tends to expand and cool. It is known since many years that an explosion can only result if the thermonuclear burning front propagates with a velocity much larger than the laminar speed of nuclear flames in degenerate matter. But only very recently it has been shown that models taking advantage of small scale turbulent velocity fluctuations lead indeed to the desired results.

In this review, recent developments in theoretical model-calculations for supernovae explosions are reviewed, including both core-collapse and thermonuclear supernovae, but with more emphasis on the (successful) thermonuclear (type Ia) models. A review concentrating on the recent progress of core-collapse supernovae is given by H.-Th. Janka in these Proceedings. 


\section{Core-collapse Supernovae}

Supernovae of type II, i.e. explosions of stars which show strong Balmer lines of hydrogen in their spectra, and also type Ib and Ic events, are thought to originate from the collapse of massive stars, $\mathrm{M} \gtrsim 8 \mathrm{M}_{\odot}$, at the end of their quiet hydrostatic nuclear burning, and the final outcome is believed to be the birth event of (most) neutron stars, and sometimes of black holes and $\gamma$-ray bursts. If this picture is correct the energy observed in the explosion ultimately must come from the binding energy of the newly born neutron star or black hole. However, although various mechanisms have been proposed which are potentially able to transform a small fraction of the gravitational energy into outward momentum of the stellar envelope, the cause of the explosion is still subject considerable discussions (see [8] for a recent review). Here we will concentrate on some micro-physics aspects of the problem and on "neutrino driven" explosions.

\subsection{Basic Input Physics}

In constructing a (core-collapse) supernova model one has to solve the hydrodynamic equations, some field equations describing gravity, rate equations for composition changes, and transport equations for particle numbers and energy for a given set of initial conditions (densities, entropies, velocities, composition variables, etc.) and material functions (equation of state, reaction rates, interaction cross-sections, etc.). It is obvious that this set of equations cannot be solved in full generality and that many approximations are necessary in order to make the problem tractable.

Concerning numerical methods for solving the hydro-equations great progress has been made during the last couple of years, provided Newtonian mechanics and gravity is a valid prescription [10]. However, in stellar collapse peak velocities approach several tenths of the velocity of light and general relativistic effects are not negligibly small, in particular at core bounce and during the early cooling and deleptonization phase of the newly born neutron star. The use of the Newtonian approximation, therefore, is questionable. For general relativistic hydrodynamics, on the other hand, the numerical techniques are much less advanced, in particular for multidimensional simulations which seem to be necessary $[3,4]$. One has to keep this situation in mind when discussing uncertainties in and implications from micro-physics input data.

\section{The Nuclear Equation of State}

One of the most important ingredients is the equation of state (EOS) and, consequently, also a major fraction of the uncertainties result from our incomplete knowledge of it. Also, there is little hope that we can learn much about the EOS from laboratory experiments, such as heavy ion collisions, 
because they test the nuclear EOS under rather different conditions. Therefore we have to rely on theoretical models or, possibly, on interpretations of astrophysical observations.

At rather low densities $\left(\varrho \lesssim 10^{-2} \varrho_{0} ; \varrho_{0} \sim 3 \times 10^{14} \mathrm{~g} \mathrm{~cm}^{-3}\right)$ the EOS can in principle be calculated from a Boltzmann-gas approximation for nucleons and nuclei, provided nuclear binding energies and partition functions are known. However, most nuclei present in the interior of a collapsing stellar core or in the outer layers of a neutron star would be very short-lived under laboratory conditions, and most of them have not even been synthesized yet by experiments. Therefore one has to rely on extrapolations from the properties of stable and "mildly" unstable nuclei.

At higher densities the Boltzmann-gas approach to the nuclear part of the EOS is no longer valid. This happens because the nuclear radius becomes comparable to the Coulomb interaction radius. Therefore, at those densities (above about $10^{12} \mathrm{~g} \mathrm{~cm}^{-3}$ ) self-consistent models have to be used, and at even higher densities, $\varrho \gtrsim 0.1 \varrho_{0}$, similar arguments show that also nucleon-nucleon interactions have to be included in such a self-consistent model. The most advanced method which has been applied to the supernova problem so far is the temperature dependent Hartree-Fock method but very little progress has been made in the past years.

Even more problematic is the EOS beyond nuclear saturation density. In the deep interior of a newly born neutron star "nuclei" dissolve into a homogeneous fluid of free neutrons and protons once the density exceeds $\varrho_{0}$. Consequently now nucleon-nucleon interactions in a dense Fermi fluid dominate the EOS. Phenomenologically determined nucleon-nucleon forces gradually lose reliability with increasing density and it is therefore not surprising that up to now the EOS at densities above, say, twice nuclear saturation density is still subject to considerable dispute, but it might be crucial for the neutrino luminosity during the first second past core-bounce, thought to trigger the ejection of the stellar envelope.

Most EOS in use in core-collapse simulations are simple, and include only part of the important physics. They are either phenomenologically motivated (e.g., [22]) or are based on mean field approaches (e.g., [21]).

\section{Weak Interaction Rates}

Next we want to mention briefly some of the uncertainties entering through our incomplete knowledge of weak interaction rates. It is well known that during most of core collapse and during the early cooling phase of newly born neutron stars typical weak interaction timescales are of the same order as the dynamic or evolution time scale of the stellar core or star, respectively. So in contrast to strong and electromagnetic interactions weak rates have to be known explicitly. Moreover, because in some cases neutrino energy distributions are not in equilibrium, it is not even sufficient to calculate energy-averaged rates. Fortunately, in this field considerable progress has 
been made recently. The best rates available to date are based on shell model wave functions or use the quasiparticle random phase approximation, but from the sensitivity of those results to details of the nuclear model one may still conclude that the calculated rates are uncertain to within a factor of two on the average, and possibly by an order of magnitude in some particular cases $[11,12]$.

Other important week interaction processes include neutrino-absorption by free neutrons, neutrino-electron scattering, neutrino-nucleus coherent scattering and neutrino-neutron scattering. These cross sections and reaction rates can, in principle, be computed numerically exactly but in most numerical studies only approximate values are used.

\section{Neutrino Transport}

During collapse and after core-bounce we will always find regions in the star where neutrinos are either streaming freely or diffusing outward. So, in principle, we have to solve the Boltzmann transport equation. This transport equation, however, is a set of complicated partial integro-differential equations and, therefore, has only recently been used in core-collapse computations, but most models still use approximations to it.

Generally speaking, neutrino transport calculations for supernovae face two major problems. Firstly, at densities below $10^{12} \mathrm{~g} \mathrm{~cm}^{-3}$ neutrinos are not in thermal equilibrium and, secondly, the diffusion approximation to the Boltzmann equation breaks down at the neutrino-sphere, where the mean free path $\bar{\lambda}$ becomes comparable to the stellar radius. The second problem is usually circumvented by introducing a so-called flux-limiter which guarantees that for $\bar{\lambda} \gg \Delta r$ the free streaming limit is obtained. The first problem can only be solved by non-equilibrium transport models such as "two-fluid models", "multi-group flux-limited" diffusion, "variable Eddington-factor" methods, Monte-Carlo transport, or even direct integrations of the Boltzmann equation $[2,9,13,16]$.

\subsection{Recent Models of Core-collapse Supernovae}

Since most of the recent numerical simulations are reviewed by Janka in these Proceedings we will not go into details but rather summarize some of the main problems.

As far as numerical simulations of stellar collapse and the subsequent supernova explosion are concerned, the most crucial phase seems to be when the newly born neutron star looses its leptons and thermal energy by neutrinos of all flavors. During this phase, both the proto-neutron star itself and the matter behind the stalled shock in the mantle are found to be unstable to entropy and/or lepton number driven buoyancy instabilities which can increase the neutrino luminosity considerably. It is commonly believed that 
this increase in luminosity is required in order to revive the stalled shock by neutrino heating [8].

Although none of the recent simulations give the desired results, namely an envelope ejection with a typical energy of about $10^{51} \mathrm{erg}$, leaving behind a neutron of about $1.4 \mathrm{M}_{\odot}$, "hope is left in Pandora's box". In fact, the simulations are close to explosions, and minor changes in the input physics and/or the numerical treatment might change the outcome. An obvious example is the neutrino luminosity of the newly-born neutron star which seems to be a bit too low for success, but can be changed during the contraction and cooling phase by different EOS's or neutrino-transport properties of hot and dense nuclear matter. A second example are hydrodynamic instabilities of the neutrino-heated convectively unstable matter behind the stalled shock which, again, can change the energy (and momentum) transport. Finally, general relativistic effects have not yet been studied in great detail in models based on realistic micro-physics input $[2,10]$.

\section{Thermonuclear Supernovae}

A strong motivation for studying thermonuclear (type Ia) supernovae comes from their use for cosmology because at present they are the most accurate cosmological distance indicators and supernovae at different redshifts are the only way to determine the nature of the suspected "dark energy" that causes the Universe to accelerate its expansion.

The most popular progenitor model for the average type Ia supernova is a massive white dwarf, consisting of carbon and oxygen, which approaches the Chandrasekhar mass $\left(\mathrm{M}_{C h} \simeq 1.4 \mathrm{M}_{\odot}\right)$ by a yet unknown mechanism, presumably accretion from a companion star, and is disrupted by a thermonuclear explosion (see [7] for a recent review). At high densities explosive carbon burning mostly leads to radioactive ${ }^{56} \mathrm{Ni}$. At lower densities intermediatemass nuclei, like ${ }^{28} \mathrm{Si}$, are produced. These elements give rise to the typical observed spectra of SNe Ia, which are dominated by lines of Fe, Si and S.

The general picture of such an explosion is that first carbon burns rather quietly in the core of the contracting white dwarf. Because this core is convectively unstable temperature fluctuations will be present and they may locally reach run-away values. After ignition, the flame is thought to propagate through the star as a sub-sonic turbulent deflagration wave which may or may not change into a detonation at low densities (around $10^{7} \mathrm{~g} \mathrm{~cm}^{-3}$ ), disrupting the star in the end in both cases.

\subsection{The Physics of Turbulent Thermonuclear Combustion}

Due to the strong temperature dependence of the carbon-fusion reaction rates nuclear burning during the explosion is confined to microscopically thin layers that propagate either conductively as subsonic deflagrations ("flames") 
or by shock compression as supersonic detonations. Both modes are hydrodynamically unstable to spatial perturbations. The best studied and probably most important hydrodynamical effect for modeling SN Ia explosions is the Rayleigh-Taylor (RT) instability resulting from the buoyancy of hot, burned fluid with respect to the dense, unburned material [14]. Subject to the RT instability, small surface perturbations grow until they form bubbles (or "mushrooms") that begin to float upward while spikes of dense fluid fall down. In the nonlinear regime, bubbles of various sizes interact and create a foamy RT mixing layer whose vertical extent grows with time. Secondary instabilities related to the velocity shear along the bubble surfaces [14] quickly lead to the production of turbulent velocity fluctuations that cascade from the size of the largest bubbles $\left(\approx 10^{7} \mathrm{~cm}\right)$ down to the microscopic Kolmogorov scale, $l_{\mathrm{k}} \approx 10^{-4} \mathrm{~cm}$, where they are dissipated. Since no computer is capable of resolving this range of scales, one has to resort to statistical or scaling approximations of those length scales that are not properly resolved. The most prominent scaling relation in turbulence research is Kolmogorov's law for the cascade of velocity fluctuations, stating that in the case of isotropy and statistical stationarity, the mean velocity $v$ of turbulent eddies with size $l$ scales as $v \sim l^{1 / 3}$.

Given the velocity of large eddies, e.g. from computer simulations, one can use this relation to extrapolate the eddy velocity distribution down to smaller scales under the assumption of isotropic, fully developed turbulence. Turbulence wrinkles and deforms the flame. These wrinkles increase the flame surface area and therefore the total energy generation rate of the turbulent front. In other words, the turbulent flame speed, defined as the mean overall propagation velocity of the turbulent flame front, becomes larger than the laminar speed. If the turbulence is sufficiently strong the turbulent flame speed becomes independent of the laminar speed, and therefore of the microphysics of burning and diffusion, and scales only with the velocity of the largest turbulent eddy [1].

As the density of the white dwarf material declines and the laminar flamelets become slower and thicker, it is plausible that at some point turbulence significantly alters the thermal flame structure [15]. So far, modeling this so-called distributed burning regime in exploding white dwarfs has not been attempted explicitely since neither nuclear burning and diffusion nor turbulent mixing can be properly described by simplified prescriptions. However, it is this regime where the transition from deflagration to detonation is assumed to happen in certain phenomenological models.

\subsection{A Numerical Model for Turbulent Combustion}

It is straight forward to convert the ideas presented in the previous section into a numerical scheme. The basic ingredients are a finite-volume method to solve the fluid-dynamics equation, a front-tracking algorithm which allows us to propagate the thermonuclear flame (assumed to be in the flamelet regime), 
and a model to determine the turbulent velocity fluctuations on unresolved sub-grid scales. Since the details of the method have been published elsewhere $[17,18,19]$ we only repeat the basic ideas here.

The central aspect of our code is a front tracking method based on a level set function $G$ which is determined in such a way that the zero level set of $G$ behaves exactly as the flame. This can be obtained from the consideration that the total velocity of the front consists of two independent contributions: it is advected by the fluid motions at a speed $\boldsymbol{v}$ and it propagates normal to itself with a burning speed $s$.

This front tracking algorithm is implemented as an additional module for the hydrodynamics code PROMETHEUS [5]. In all simulations presented here a simple implementation was used which, however, describes the basic physics quite well $[17,18]$. It assumes that the $G$-function is advected by the fluid motions and by burning and is only used to determine the source terms for the reactive Euler equations. Nuclear burning can now be computed provided the normal velocity of the burning front is known everywhere and at all times. In computations discussed in the following it is determined according to a flame-brush model of [14].

\subsection{Some Results of Supernova Simulations}

We have carried out numerical simulations in $2 \mathrm{D}$ and $3 \mathrm{D}$, for a variety of different initial conditions, and for different numerical resolution. In most of our simulations the white dwarf, constructed in hydrostatic equilibrium for a realistic equation of state, has a central density of $2.9 \times 10^{9} \mathrm{~g} \mathrm{~cm}^{-3}$, a radius of $1.5 \times 10^{8} \mathrm{~cm}$, and a mass of $2.8 \times 10^{33} \mathrm{~g}$, identical to the one used in [14]. The initial mass fractions of $\mathrm{C}$ and $\mathrm{O}$ are chosen to be equal, and the total binding energy is $5.4 \times 10^{50} \mathrm{erg}$. At low densities $\left(\rho \leq 10^{7} \mathrm{~g} \mathrm{~cm}^{-3}\right)$, the burning velocity of the front is set equal to zero because the flame enters the distributed regime and our physical model is no longer valid. However, since in reality some matter may still burn the energy release obtained in the simulations is probably somewhat too low. An extended parameter study, varying the chemical composition as well as the ignition density, is presently under way and will be published elsewhere.

A first and important result is that we do find numerically converged solutions. Although an increase in spatial resolution gives more structured burning fronts with larger surface area, the corresponding increase of fuel consumption is compensated by the lower values of the turbulent velocity fluctuations on smaller length scales. So the net effect is that, for identical initial conditions, the explosion energies are independent of the numerical resolution, demonstrating that the level-set prescription allows one to resolve the structure of the burning front down almost to the grid scale, thus avoiding artificial smearing of the front, which is an inherent problem of front-capturing schemes. 


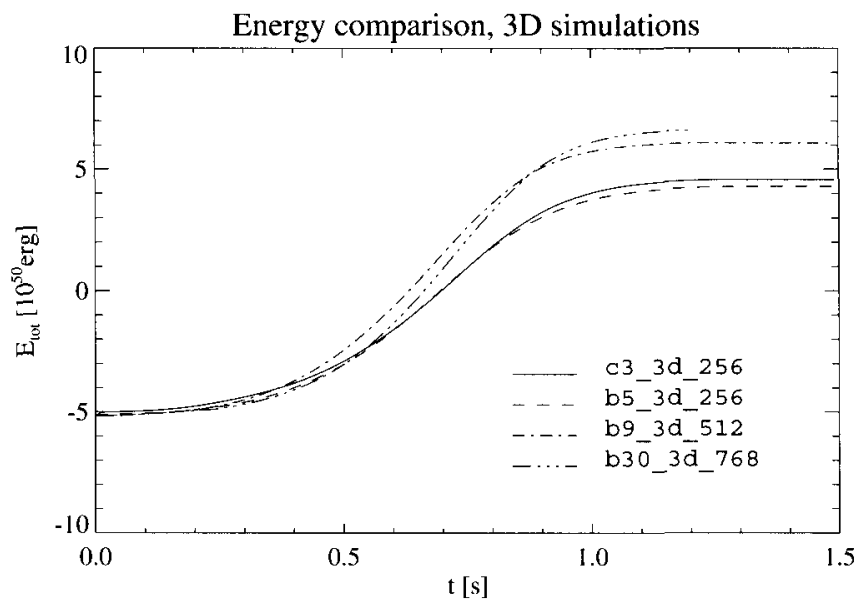

Fig. 1. Energy evolution of several three-dimensional (3d) explosion models (dashed and dashed-dotted). For comparison we also show a centrally ignited ("three fingers") model of [18] (solid line). The other labels give the number of initial "bubbles" (bn) and the number of grid points per dimension (ijk).

In our approach, the initial white dwarf model (composition, central density, and velocity structure), as well as assumptions about the location, size and shape of the flame surface as it first forms, fully determine the simulation results. A plausible ignition scenario suggested by $[6]$ is the simultaneous runaway at several different spots in the central region of the progenitor star. Therefore, in the following we will concentrate on such initial conditions. Fig. 1 shows the energy generated for a series of models including, for comparison, one centrally ignited model (3c_3d_256).

During the first 0.5 seconds, all models are nearly indistinguishable as far as the total energy is concerned, which at first glance appears somewhat surprising, given the quite different initial conditions. A closer look at the energy generation rate actually reveals noticeable differences in the intensity of thermonuclear burning for the simulations, but since the total flame surface is initially very small, these differences have no visible impact on the integrated curve in the early stages.

However, after about 0.5 seconds, when fast energy generation sets in, the models with more ignition spots burn more vigorously due to their larger surface and therefore they reach higher final energy levels. Fig. 1 also shows that the centrally ignited model (c3_3d_256) is almost identical to the off-center model b5_3d_256 with regard to the explosion energetics. But, obviously, the scatter in the final energies due to different initial conditions appears to be small. Moreover, all models explode with an explosion energy in the range of what is observed. 
Table 1. Overview over element production and energy release of typical supernova simulations

\begin{tabular}{lccc}
\hline model & $m$ "Mg" $\left[M_{\odot}\right]$ & $m$ "Ni" $\left[M_{\odot}\right]$ & $E_{\text {nuc }}\left[10^{50} \mathrm{erg}\right]$ \\
\hline c3_3d_256 & 0.177 & 0.526 & 9.76 \\
b5_3d_256 & 0.180 & 0.506 & 9.47 \\
b9_3d_512 & 0.190 & 0.616 & 11.26 \\
\hline
\end{tabular}

\subsection{Predictions for Observable Quantities}

In this section we present a few results for various quantities which can, in principle, be observed and which therefore can serve as tests for the models.

The most direct test of explosion models is provided by observed light curves and spectra. According to "Arnett's Law" light curves measure mostly the amount and spatial distribution of radioactive ${ }^{56} \mathrm{Ni}$ in type Ia supernovae, and spectra measure the chemical composition in real and velocity space.

In [20] the results of one of our centrally ignited 3D-models have been used, averaged over spherical shells, to compute color light curves in the UBVI-bands. Their code assumes LTE radiation transport and loses reliability at later times (about 4 weeks past maximum) when the supernova enters the nebular phase. Also, this assumption and the fact that the opacity is not well determined at longer wavelength make I-light curves less accurate. Keeping this in mind, they produce the light curves of typical SNe Ia very well. The main reason for the good agreement between the model and, e.g., SN 1994D is the presence of high-velocity radioactive $\mathrm{Ni}$ in outer layers of the supernova model which is not be predicted by spherical models.

A summary of the gross abundances obtained for some of our 3D models is given in Table 1. Here "Mg" stands for intermediate-mass nuclei, and "Ni" for the iron-group. In addition, the total energy liberated by nuclear burning is given. Since the binding energy of the white dwarf was about $5 \times 10^{50} \mathrm{erg}$, all models do explode. Typically one expects that around $80 \%$ of iron-group nuclei are originally present as ${ }^{56} \mathrm{Ni}$ bringing our results well into the range of observed Ni-masses. This success of the models was obtained without introducing any non-physical parameters, but just on the basis of a physical and numerical model of subsonic turbulent combustion. We also stress that our models give clear evidence that the often postulated deflagration-detonation transition is not needed to produce sufficiently powerful explosions.

Finally, we have "post-processed" several of our models in order to see whether or not also reasonable isotopic abundances are obtained. The results, shown in Fig. 2, are preliminary and should be considered with care. However, it is obvious that, with a few exceptions, also isotopic abundances are within the expected range. Exceptions include the high abundance of (unburned) $\mathrm{C}$ and $\mathrm{O}$, and the overproduction of ${ }^{48,50} \mathrm{Ti},{ }^{54} \mathrm{Fe}$, and ${ }^{58} \mathrm{Ni}$. We think that this reflects a deficiency of some of our models which burn to little $\mathrm{C}$ and $\mathrm{O}$ at 


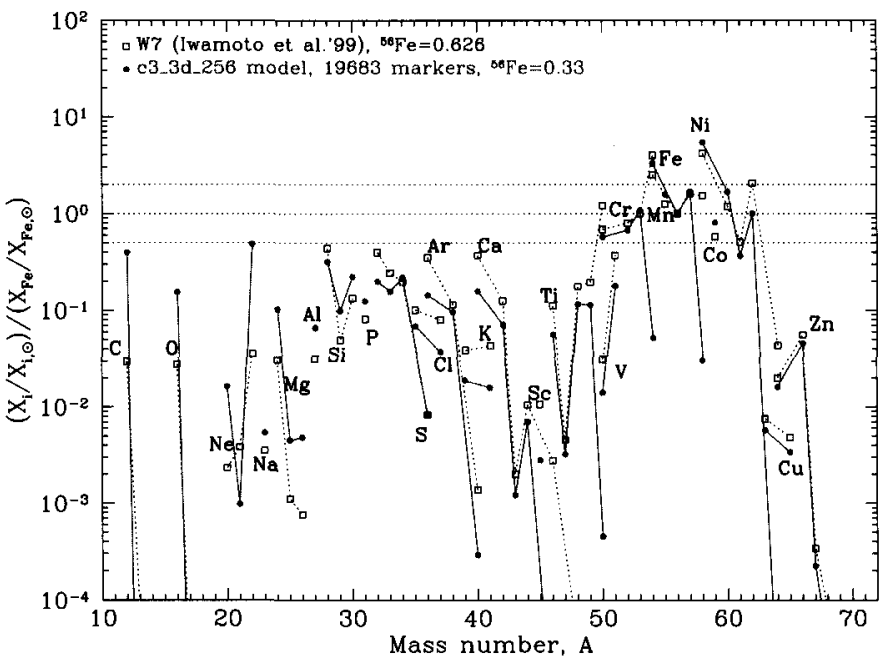

Fig. 2. Isotopic abundances obtained for the centrally ignited $3 \mathrm{D}$ model $3 \mathrm{c} \_3 \mathrm{~d} \_256$ in comparison to $\mathrm{W} 7$ predictions (Travaglio et al., in preparation).

densities too high and temperatures too low, and which also, because of the low ${ }^{56} \mathrm{Ni}$, would not give a good light curve.

\section{Summary and Conclusions}

The physics of supernovae as well as the present status of numerical simulations of the explosion process have been discussed. It was demonstrated that multi-dimensional models are needed for both types and that, to a certain extent, they have been carried out in $2 \mathrm{D}$ with moderately good physics input for core-collapse supernovae, and in $3 \mathrm{D}$ with just sufficient numerical resolution for thermonuclear ones. The bad news is that these calculations have not solved the core-collapse supernova problem yet, and the limiting factor does not seem to be the numerical resolution of the simulations but rather still existing uncertainties in the (micro-) physics. This is in contrast to thermonuclear explosion models where the physics is reasonably well under control, but the numerical resolution is barely sufficient.

In case of core collapse supernovae it is likely that with the next generation of supercomputers one will be able to carry out fully resolved simulations in $3 \mathrm{D}$, but most of the work has to go into improving the micro-physics and the neutrino transport. In contrast, for thermonuclear explosions one will never be able to resolve all relevant scales numerically and, therefore, developing new and clever tools to overcome this difficulty is a must. However, even the recent (admittedly still limited) type Ia models do predict explosion energies, 
light curves, spectra, and nuclear abundances that are well within the range of their observed counterparts.

\section{References}

1. P. Clavin: Ann. Rev. Fluid Mech. 26, 321 (1994)

2. R. Buras et al. : Phys. Rev. Lett. 90, 1101 (2003)

3. H. Dimmelmeier, J.A. Font, E. Müller: Astron. Astrophys. 388, 917 (2003)

4. H. Dimmelmeier, J.A. Font, E. Müller: Astron. Astrophys. 393, 523 (2003)

5. B.A. Fryxell, E. Müller, W.D. Arnett: MPA Preprint 449 (1989)

6. D. Garcia-Senz, S.E. Woosley: Astrophys. J. 454, 895 (1995)

7. W. Hillebrandt, J.C. Niemeyer: Ann. Rev. Astron. Astrophys. 38, 191 (2000)

8. H.-Th. Janka et al. : Core Collapse and Then? The Route to Massive Star Explosions. In: From Twilight to Highlight: The Physics of Supernovae, ed by W. Hillebrandt, B. Leibundgut (Springer, Berlin Heidelberg New York 2003) p. 39

9. M.Th. Keil, G.G. Raffelt, H.-Th. Janka: Astrophys. J. 590, 971 (2003)

10. K. Kifonidis et al. : Astron. Astrophys. 408, 621 (2003)

11. K. Langanke, G. Martinez-Pinedo: Rev. Mod. Phys. 75, 819 (2003)

12. K. Langanke et al. : Phys. Rev. Lett. 90, 1102 (2003)

13. M. Liebendörfer et al. : Nuc. Phys. A719, 144 (2003)

14. J.C. Niemeyer, W. Hillebrandt: Astrophys. J. 452, 769 (1995)

15. J.C. Niemeyer, S.E. Woosley: Astrophys. J. 475, 740 (1997)

16. M. Rampp, H.-Th. Janka: Astron. Astrophys. 396, 361 (2002)

17. M. Reinecke, W. Hillebrandt, J.C. Niemeyer: Astron. Astrophys. 347, 739 (1999)

18. M. Reinecke, W. Hillebrandt, J.C. Niemeyer: Astron. Astrophys. 386, $936(2002)$

19. M. Reinecke, W. Hillebrandt, J.C. Niemeyer: Astron. Astrophys. 391, 1167 (2002)

20. E. Sorokina, S. Blinnikov: Light Curves of Type Ia Supernovae as a Probe for an Explosion Model. In: From Twilight to Highlight: The Physics of Supernovae, ed by W. Hillebrandt, B. Leibundgut (Springer, Berlin Heidelberg New York 2003) p. 268

21. K. Sumiyoshi, H. Toki: Astrophys. J. 422, 7001994

22. F.D. Swesty, J.M. Lattimer, E.S. Myra: Astrophys. J. 425, 195 (1994) 